\title{
Estrutura Fatorial da Escala de Atitudes Frente a Relacionamentos Afetivos Estáveis
}

\author{
Taciano Lemos Milfont \\ Universidade Federal de Alagoas \\ Valdiney Veloso Gouveia \\ Universidade Federal da Paraíba \\ Girlene Ribeiro de Jesus \\ Universidade de Brasília ${ }^{1}$ \\ Estefânea Élida da Silva Gusmão \\ Universidade Federal de Pernambuco \\ Sandra S. da Silva Chaves \\ Jorge Artur Peçanha de Miranda Coelho \\ Universidade Federal da Paraíba
}

\begin{abstract}
RESUMO - O presente estudo visa contribuir para a comprovação da estrutura fatorial da Escala de Atitudes Frente a Relacionamentos Afetivos Estáveis (RAE; Reis, 1995). Participaram da pesquisa 658 estudantes universitários com idade média 19,9 anos $(D P=2,5)$. Análises fatoriais exploratórias e confirmatórias indicaram a existência de uma estrutura multidimensional. Os cinco fatores encontrados (Comprometimento, Edificação, Mutualidade, Envolvimento e Indulgência) apresentaram índices de consistência interna satisfatórios $(0,68<\alpha<0,83)$, itens com saturações altas $\left(0,46<\lambda_{\mathbf{y}}<0,75\right)$ e significativas $(p<0,05)$. Esta estrutura multidimensional apresentou melhores índices de bondade de ajuste $\left(\chi^{2} / g l=3,07 ; \mathrm{y} F F I=0,91 ; C F I=0,97 ; R M S E A=0,056\right)$ e foi estatisticamente superior $\left[\Delta \chi^{2}(111)=676,69, p<0,001\right]$ à estrutura unidimensional. Resultados de correlações com as auto-imagens independente e interdependente (Singelis, 1994), com os valores humanos básicos (Gouveia, 2003), além de diferenças quanto ao sexo, confirmam a validade dos cinco novos fatores.
\end{abstract}

Palavras-chave: atração interpessoal; relação afetiva estável; escala ERA; análise fatorial.

\section{Factor Structure of the Stable Affective Relationships Scale}

\begin{abstract}
This article investigates the factor structure of the Stable Affective Relationships Scale (SAR; Reis, 1995). Exploratory and confirmatory factor analyses of data from 658 Brazilian undergraduate students with average age 19.9 years old $(S D=2.5)$, are reported. These analyses revealed five factors underlying the items on the SAR (Commitment, Construction, Mutuality, Involvement, and Indulgence) that are important in a stable affective relationship. All five factors presented good reliability $(.68<\alpha<.83)$, items with high $(.46<\lambda<.75)$ and significant $(p<.05)$ factor loadings. This five-factor structure had a better fit to the data $\left(\chi^{2} / d f=3.07 ; G F I=.91 ; C F I=.97 ; R M S E A=.056\right)$ and was statistically better fitting $\left[\Delta \chi^{2}(111)=676.69\right.$, $p<.001]$ than the unidimensional structure. The results also provided support for the validity of these new factors in exploring correlation with independent and interdependent self-construals (Singelis, 1994), basic human values (Gouveia, 2003) and gender differences.
\end{abstract}

Key words: interpersonal attraction; stable affective relationships; SAR scale; factor analysis.

A atração interpessoal é um tema freqüente em Psicologia Social, com capítulos específicos de manuais da área dedicados ao tema. Por exemplo, o manual mais conhecido desta disciplina na Europa (Hewstone \& Strobe, 2001) e o mais vendido nos Estados Unidos (Baron \& Byrne, 2002), segundo Smith e Bond (1993), dedicam capítulos ao tema. $\mathrm{O}$ mesmo ocorrendo com manuais nacionais (Rodrigues, Assmar \& Jablonski, 2001) e traduções (Aronson, Arket \& Wilson, 2002; Myers, 2000).

Estudos de meta-análises também corroboram sua importância. Em recente revisão de meta-análises em Psicologia Social, Richard, Bond e Stokes-Zoota (2003) incluíram a atração interpessoal (denominado por eles de relationship) entre

Endereço: SQN 212, Bloco A, Ap. 509, Asa Norte, Brasília, DF, Brasil 70864-010.E-mail: girleneribeiro@yahoo.com.br um dos 18 tópicos pesquisados ${ }^{2}$. A partir de 32 conclusões baseadas em meta-análises prévias, eles verificaram que as relações interpessoais apresentam um dos maiores índices de efeito ( $r=0,22$; a média geral do efeito de todos os 18 tópicos foi de 0,21 ). Entre as variáveis pesquisadas neste tópico, as cinco que apresentaram maiores efeitos foram: mulheres tendem a ser mais atraídas por homens mais altos, enquanto homens o são por mulheres mais baixas $(r=0,46)$, mulheres fisicamente atraentes são populares $(r=0,40)$, homens fisica-

2 Os tópicos pesquisados com a relativa média do efeito foram: agressão $(r=0,24)$, atitudes $(r=0,27)$, atribuição $(r=0,14)$, efeitos de expectativa $(r=0,16)$, papéis sexuais $(r=0,18)$, processos grupais $(r=0,32)$, psicologia hospitalar $(r=0,17)$, comportamentos de ajuda $(r=0,18)$, relações intergrupais $(r=0,19)$, lei $(r=0,17)$, liderança $(r=0,25)$, metodologia $(r=0,21)$, motivação $(r=0,15)$, comunicação não-verbal $(r=0,22)$, personalidade $(r=0,21)$, relações interpessoais $(r=0,22)$, cognição social $(r=0,20)$ e influência social $(r=0,13)$. 
mente atraentes são populares $(r=0,36)$, as pessoas tendem a se revelar para as pessoas de quem gostam $(r=0,34)$ e a atração interpessoal tende a ser recíproca $(r=0,22)$.

Nesse contexto, poder-se-ia dizer que a atração interpessoal constitui um tema clássico em Psicologia Social, havendo muitas abordagens teóricas e medidas psicológicas a ele relacionadas. $\mathrm{O}$ presente estudo tenta contribuir para a comprovação da estrutura fatorial de uma dessas medidas, a Escala de Atitudes Frente a Relacionamentos Afetivos Estáveis (RAE; Reis, 1995). Antes de considerá-la mais detalhadamente, demanda-se, porém, uma revisão de algumas das principais abordagens teóricas e medidas existentes.

\section{Atração Interpessoal: Abordagens Teóricas e Instrumentos de Medida}

As relações interpessoais têm sido diferenciadas entre relações de amizade e de amor (Alferes, 1996) e têm sido estudadas por meio de teorias de organização social - tais como o princípio de equilíbrio de Heider, teoria de comunicação social informal e comparação social de Festinger e força em direção à simetria de Newcomb -, além de teorias do reforço e troca social (ver Alferes, 1996; Rodrigues, Assmar \& Jablonski, 2001). No entanto, existem fatores que são comuns aos vários tipos de atração: beleza física, proximidade física, semelhanças em atitudes e valores, e avaliações positivas (Vaughan \& Hogg, 2002).

Um aspecto das relações interpessoais que tem recebido maior atenção é o amor, pois ele é de grande importância na vida dos indivíduos, tanto no aspecto pessoal como social (García, 2000). Segundo García (2000), o estudo do amor é uma das áreas de maior relevância na Psicologia Social. Ubillos e cols. (1997), por exemplo, argumentam que cada vez mais o amor é considerado como um fenômeno universal por psicólogos, antropólogos e outros estudiosos, mesmo que seu significado possa variar completamente de uma cultura a outra e também em épocas diferentes.

Várias definições de amor têm sido apresentadas. Reis (1992) destaca a definição de Rubin (1970) que interpreta o amor como uma "atitude mantida por uma pessoa em relação a uma outra pessoa particular, a qual envolve predisposições para pensar, sentir e comportar-se de determinadas maneiras relativamente àquela pessoa" (p. 265). Bystronski (1991) afirma que os estudos de Rubin (1970) foram marcos iniciais na tentativa de construção de teorias acerca do amor, na medida em que fez uma distinção entre o amor e o gostar.

Assim, diversas abordagens teóricas acerca do amor foram criadas, tais como As Cores do Amor de Lee (1977) e a Teoria Triangular do Amor de Stenberg (1986). Lee (1977) apresenta vários estilos de amor, divididos em primários, secundários e terciários. Estilos primários são: eros (amor passional), ludus (amor como um jogo) e storge (amor amigo). Os estilos secundários são: pragma (amor racional, lógico), mania (amor possessivo, dependente) e ágape (amor divino, altruísta). Por fim, os estilos terciários são combinações dos dois primeiros: eros lúdico, eros estórgico e ludus_estórgico. Stenberg (1986), por sua vez, propõe a existência de três componentes essenciais ao fenômeno do amor: intimidade, paixão e decisão/comprometimento (para uma revisão mais detalhada das teorias do amor, ver Hernandez \& Oliveira, 2003; Reis, 1992).
A partir das abordagens teóricas, várias escalas de mensuração do amor foram criadas. Rubin (1970) desenvolveu duas escalas para medir os construtos amar e gostar; Hendrick e Hendrick (1986) desenvolveram a Love Attitudes Scale, baseados nos estilos de amor de Lee (1977); e Stenberg (1997) criou a Triangular Love Scale a partir de sua própria teoria. Muitas destas escalas têm sido traduzidas e utilizadas no Brasil (ver, por exemplo, Bystronski, 1991; Hernandez \& Oliveira, 2003). Não obstante, este procedimento é considerado um embargo ético (Smith \& Bond, 1993), pois assume que as situações relacionadas à atração interpessoal encontradas em outros países têm o mesmo significado para a população brasileira. Desta forma, é mais recomendada a utilização de instrumentos desenvolvidos no e para um contexto específico; e já existem algumas medidas criadas no contexto brasileiro relacionadas ao tema da atração interpessoal. Ramos, Yazawa e Salazar (1994) contruíram a Escala de Ciúme Romântico (ver, também, Belo, Raymundo, Gouveia, Milfont \& Vasconcelos, 2002), e Reis (1995) propôs a Escala de Atitudes Frente a Relacionamentos Afetivos Estáveis (RAE). Esta última compreende o foco principal do presente artigo.

\section{Escala de Atitudes frente a Relações Afetivas Estáveis (RAE)}

Reis (1995) desenvolveu a escala RAE com o objetivo de medir atitudes frente a relações afetivas estáveis, e verificar a influência do sexo e estado civil nas atitudes frente a esse tipo de relacionamento íntimo. Os itens da escala foram construídos a partir de entrevistas com adolescentes e adultos. A seleção dos items foi realizada em duas etapas. Na primeira etapa os itens iniciais foram administrados a 100 estudantes universitários que indicavaram a importância que atribuíam a cada item para uma relação afetiva estável. Os itens que apresentaram $80 \%$ de concordância acerca de sua importância nessa etapa do estudo foram mantidos. Na segunda etapa, os 30 itens selecionados foram administrados em um pré-teste com 111 estudantes universitários, confirmando a adequação de todos os itens. Após a fase de seleção dos itens, a fidedignidade da escala foi testada com uma amostra de univertários brasileiros $(N=257)$ e outra de universitários estadunidenses $(N=167)$. A escala apresentou alta fidedignidade em ambas as amostras $(\alpha=0,91$ e $\alpha=0,89$, para os universitários brasileiros e estadunidenses, respectivamente). A validade da escala foi testada por meio da correlação com as seis subscalas da Love Attitudes Scale, de Hendrick e Hendrick (1986). A RAE apresentou correlações significativas e positivas com as subscalas eros, mania, pragma e ágape, e correlação negativa com a subscala ludus. A subscala storge não apresentou correlação significativa.

Corroborando o teoricamente esperado, a autora verificou diferenças significativas nos seguintes aspectos: as mulheres e as pessoas que namoravam apresentaram maiores escores na RAE do que os homens e as que não namoravam. Ainda, as pessoas casadas apresentaram maiores escores na RAE do que as não casadas, mas que pretendiam se casar, e, estas últimas, apresentaram maiores escores do que as que não pretendiam se casar. A autora também realizou uma Análise dos Componentes Principais (ACP) sem fixar o número de componentes. Os resultados indicaram a existência de oito 
componentes com eingenvalues acima de 1,00, os dois primeiros com eingenvalues acima de 3,0 que juntos explicaram $64,9 \%$ da variância total. A partir destes resultados inicias, a autora realizou nova ACP fixando como critério um eingenvalue igual ou superior a 3,00. Após esta segunda ACP, a autora utiliza a escala RAE como unidimensional.

Os resultados apresentados acima confirmam a validade psicométrica da RAE. Todavia, não há uma clareza quanto a sua estrutura fatorial. A autora verifica a possibilidade de uma estrutura bidimensional, com os fatores Amor Romântico e Amizade (ver Bystronski, 1991; Reis, 1995), porém não apresenta os parâmetros de fatorabilidade da matriz de correlações (KMO, Teste de Esfericidade de Bartlett), os procedimentos utilizados na análise (tipo de rotação adotada, critério de inclusão dos fatores), tampouco foi apresentada a estrutura fatorial final (itens de cada fator com suas variâncias explicadas e respectivos índices de consistência interna) e a intercorrelação entre os fatores. Ademais, a própria autora, contrariando os seus resultados sobre a estrutura fatorial desta medida, utiliza a escala como unidimensional.

Diante do exposto, o objetivo principal deste estudo foi apresentar maiores evidências sobre a validade e precisão de uma escala relacionada ao tema da atração interpessoal desenvolvida no Brasil. Assim, o objetivo foi testar a estrutura fatorial (validade de construto) da RAE, além de conhecer sua validade convergente-discriminante e preditiva. Neste caso, procurou-se verificar se os participantes do sexo feminino e as pessoas que possuem um relacionamento afetivo duradouro apresentam maiores pontuações na RAE do que aqueles do sexo masculino e que estavam solteiros no momento da pesquisa. Finalmente, objetivou-se ainda conhecer sua precisão (fidedignidade, consistência interna).

\section{Método}

\section{Amostra}

Participaram desta pesquisa 658 estudantes universitários recém-ingressos de uma instituição de ensino pública e outra particular. Todos eram solteiros, provenientes de cursos das três grandes áreas do conhecimento (Exatas, Humanas e Saúde), com a maior concentração de alunos de Administração (19,5\%) para a instituição pública, e Fonoaudiologia $(22,8 \%)$ para a particular. A amostra foi praticamente eqüitativamente distribuída quanto ao sexo (Homens, $n=337$; Mulheres, $n=321)$, sendo a maioria católica $(69,8 \%)$. A idade média foi de aproximadamente 20 anos, tendo o mais jovem 17 e o mais velho 42 anos; $33,9 \%$ se classificaram como de classe média e apenas alguns poucos se classificaram nos extremos, como de classe baixa $(0,2 \%)$ ou alta $(0,9 \%)$.

\section{Instrumentos}

Além de questões sociodemográficas, os participantes responderam aos seguintes instrumentos:

- Escala de Atitudes Frente a Relações Afetivas Estáveis (RAE) (Reis, 1995). Esta medida é composta por 30 itens, respondidos em uma escala de cinco pontos, tipo Likert, indo de 1 (Absolutamente Dispensável) a 5 (Absolutamente Indispensável). Tem por objetivo veri- ficar as atitudes favoráveis a relações afetivas estáveis e foi validada no Brasil ( $n=329)$ e nos Estados Unidos da América $(n=167)$, obtendo alta fidedignidade em ambas as amostras ( $\alpha=0,91$ e 0,89 , respectivamente).

- Escala de Auto-Imagem (EAI) (Gouveia, Singelis \& Coelho, 2002; Singelis, 1994). Foi utilizada a versão completa com 30 itens (ver Milfont, no prelo), igualmente distribuídos entre as duas auto-imagens: Independente (por exemplo, Gosto de ser único e diferente das outras pessoas em muitos aspectos; Faço minhas próprias coisas, independentemente do que pensam as outras pessoas) e Interdependente (por exemplo, Sacrificarei meu próprio interesse em benefício do grupo em que estou; Sinto que meu destino se mistura com o destino daqueles que me rodeiam). A EAI tem o propósito de medir o conjunto de pensamentos, sentimentos e ações que compõem as duas auto-imagens. Todos os 30 itens apresentam a mesma direção e os respondentes expressam seu grau de concordância utilizando uma escala de sete pontos, tipo Likert, variando de 1 (Discordo Fortemente) a 7 (Concordo Fortemente).

- Questionário dos Valores Básicos (Gouveia, 1998, 2003). É composto por 24 valores básicos, e os respondentes expressam o grau de importância de cada valor como princípio-guia em suas vidas através de uma escala de sete pontos, indo de 1 (Nada Importante) a 7 (Muito Importante). Os valores foram computados para formar os três critérios de orientação: Pessoal (emoção, sexual, prazer, estimulação, êxito, poder, prestígio, autodireção e privacidade), Central (justiça social, beleza, maturidade, sabedoria $^{3}$, saúde, estabilidade pessoal e sobrevivência) e Social (convivência, honestidade, apoio social, afetividade, religiosidade, obediência, tradição e ordem social).

\section{Procedimento}

A coleta dos dados foi realizada por um único pesquisador, que seguiu um procedimento padrão. Foram passados para os participantes os esclarecimentos sobre o anonimato e sigilo das respostas e, obtendo o consentimento verbal, os participantes foram orientados quanto ao preenchimento do questionário. Garantiu-se o caráter voluntário da participação, bem como o respeito às diretrizes éticas que regem a pesquisa com seres humanos. Pediu-se para que as respostas fossem dadas individualmente e que não fosse deixado nenhum item em branco. Os questionários foram respondidos individualmente, mas em contexto coletivo de sala de aula. Os instrumentos foram contrabalançados, para evitar o efeito de exposição da informação e possível viés de resposta.

\section{Análise dos dados}

As análises descritivas (frequiências, medidas de tendência central e dispersão), correlações $r$ de Pearson, análise de variância (ANOVA), testes $t$ de Student, Análise Fatorial Exploratória (AFE) e o cálculo da consistência interna (Alfa de Cronbach) foram realizadas por meio do pacote estatístico Statistical Package for the Social Sciences (SPSS), versão 11.5. A Análise Fatorial Confirmatória (AFC) foi realizada com a

3 Atualmente denominado conhecimento (ver Gouveia, 2003). 
versão 8 do programa LISREL (Jöreskog \& Sörbom, 1993). Decidiu-se realizar este tipo de análise porque ela permite testar hipóteses específicas sobre a estrutura latente do modelo (por exemplo, número de fatores, cargas fatoriais), apresentando os respectivos indicadores de bondade de ajuste do modelo. A técnica de Imputação Múltipla (Multiple Imputation) (Jöreskog \& Sörbom, 1996; Schafer, 1997) foi utilizada para substituir os casos faltosos (missing values). Estes casos faltosos foram inferiores a $5 \%$ em todas as variáveis. A técnica de Imputação Múltipla é correntemente implementada em Psicologia (Schafer \& Graham, 2002; Sinharay, Stern \& Russell, 2001).

Foram utilizados os seguintes índices de bondade de ajuste: a razão do qui-quadrado pelo grau de iberdade $\left(\chi^{2} / g . l\right.$.), o índice de bondade de ajuste (Goodness-of-Fit Index, GFI), o índice de ajuste comparativo (Comparative Fit Index, CFI), a raíz quadrada média do erro de aproximação (Root Mean Square Error of Approximation, RMSEA) com o seu intervalo de confiança de 90\% (90 Percent Confidence Interval for RMSEA), e a raíz quadrada média residual padronizada (Standardized Root Mean Square Residual, SRMR). Recomenda-se valores do $\chi^{2} / g$.l. entre 2,00 e 3,00 (Carmines \& McIver, 1981). Para a verificação da adequação do modelo aos dados também é recomendado valores do GFI e CFI superiores ou próximos a 0,90 e 0,95, respectivamente (Garson, 2003; Hu \& Bentler, 1999). Com relação aos valores de RMSEA e SRMR, recomendam-se valores próximos a 0,06 e 0,08, respectivamente (Hu \& Bentler, 1999).

Outros três indicadores foram utilizados para seleção de modelos concorrentes: o teste da diferença do qui-quadrado (Chi-Square Difference Test), o índice esperado de validação cruzada (Expected Cross-Validation Index, ECVI) com o seu intervalo de confiança de $90 \%$ (90 Percent Confidence Interval for ECVI) e o critério de informação consistente de Akaike (Consistent Akaike Information Criterion, CAIC). Resultados estatisticamente significativos no teste da diferença do qui-quadrado, e menores valores no ECVI e CAIC indicam o modelo que melhor se adequa aos dados (Garson, 2003).

\section{Resultados}

\section{Análise Fatorial Exploratória (AFE)}

Inicialmente verificou-se a fatorabilidade da matriz de correlações dos 30 itens da escala RAE, a qual se apresentou adequada: Kaiser-Meyer-Olkin (KMO) $=0,94$ e o Teste de Esfericidade de Bartlett $=\chi^{2}(435)=7.488,07, p<0,001$. Como o objetivo foi identificar possíveis construtos teóricos latentes, foi realizada uma Análise Fatorial Exploratória (AFE), adotando o método de extração dos eixos principais (PAF) e rotação promax (Fabrigar, MacCallum, Wegener \& Strahan, 1999). Para verificar o número de fatores a serem retidos, utilizou-se o critério de eigenvalue maior do que 1,00, o teste scree e o critério de análise paralela (Laros, 2004).

Os resultados da AFE indicaram a existência de cinco fatores com eigenvalues maiores que 1,0. O teste scree suporta esta solução, com uma susbstancial quebra no gráfico após o quinto fator (os 10 primeiros eigenvalues foram: 9,95, 1,94, 1,45, 1,25, 1,19, 0,94, 0,87, 0,81, 0,80 e 0,77). O critério de análise paralela baseada nos eigenvalues da AFE e em 100 matrizes de correlações randômicas (O'Connor, 2000), indicou que os cinco primeiros eigenvalues obtidos empiricamente $(9,39,1,44,0,85$, 0,68 e 0,62 ) foram superiores aos cinco obtidos randomicamente $(0,47,0,40,0,36,0,32$ e 0,29). Conjuntamente, estes resultados indicam a extração de cinco fatores, que explicaram 52,6\% da variância total. A Tabela 1 apresenta os resultados.

Como pode ser visto, os itens 13, 15, 18, 23 e 28 apresentaram saturação inferior a 0,30 ou saturação igual ou superior a 0,30 em dois ou mais fatores, sendo excluídos nas análises estatísticas subsequientes. Os cinco fatores encontrados a partir da análise fatorial podem ser descritos como segue:

Fator I. Foi composto por seis itens com saturações variando entre 0,32 (diálogo) e 0,84 (confiança). Apresentou eigenvalue igual a 9,95, explicando $33,1 \%$ da variância total e com índice de consistência interna $(\alpha)$ igual a 0,83 . Por ser composto por itens que sugerem compromisso com a relação afetiva (confiança, sinceridade, fidelidade, respeito, amor e diálogo), foi denominado de Comprometimento.

Fator II. Apresentou eigenvalue igual a 1,94, explicando $4,8 \%$ da variância total. Formado por sete itens, com saturações entre 0,35 (ausência de egoísmo) e 0,63 (conhecimento das intenções do outro), e com consistência interna de 0,77 , foi denominado de Edificação. O conteúdo dos seus itens sugere aspectos importantes na construção de uma relação afetiva estável (conhecimento das intenções do outro, coerência entre palavras e atos de parte a parte, crédito de parte a parte à relação, preocupação consigo e com o outro, capacidade de lidar com os problemas, intimidade e ausência de egoísmo).

Fator III. Quatro itens formaram este fator, apresentando saturações que variaram entre 0,50 (disposição mútua para enfrentar as dificuldades juntos) e 0,76 (cooperação mútua). Apresentou eigenvalue igual a 1,45 , explicando $2,8 \%$ da variância total. Seu índice de consistência interna foi de 0,77 , tendo sido denominado de Mutualidade por englobar aspectos relativos a reciprocidade de objetivos e sentimentos (cooperação mútua, construção compartilhada de algo, planos compartilhados e disposição mútua para enfrentar as dificuldades juntos).

Fator IV. Composto por cinco itens, com saturações variando de 0,35 (estar juntos em eventos importantes da vida de cada um) a 0,63 (sentir a falta na ausência do outro) e com eigenvalue de 1,25, este fator explicou $2,3 \%$ da variância total. Denominado de Envolvimento, possui itens que contemplam tal aspecto (sentir a falta na ausência do outro, externalização freqüente dos sentimentos de parte a parte, encontros freqüentes e regulares, real envolvimento afetivo, ou vontade de se envolver e estar juntos em eventos importantes da vida de cada um). Seu índice de consistência interna foi de 0,71 .

Fator $V$. Este último fator foi composto por três itens, com saturações variando entre 0,48 (compreensão mútua) e 0,63 (aceitação). Apresentou eigenvalue de 1,19, sendo responsável pela explicação de $2,1 \%$ da variância total. Seu índice de consistência interna foi de 0,68 , tendo sido denominado de Indulgência, pois contempla itens referentes ao respeito ao outro (aceitação, tolerância e compreensão mútua).

\section{Análise Fatorial Confirmatória (AFC)}

Foram realizadas AFCs utilizando a matriz de covariância e com o método de Máxima Verossimilhança. Em razão de não serem conhecidos os itens da possível estrutura bidimen- 
Tabela 1. Estrutura Fatorial da Escala de Atitudes Frente a Relações Afetivas Estáveis (RAE).

\begin{tabular}{|c|c|c|c|c|c|c|}
\hline Itens & $\mathbf{I}$ & II & III & IV & $\mathbf{V}$ & $\underline{\mathbf{h}}^{2}$ \\
\hline 01. Confiança & 0,84 & 0,12 & $-0,12$ & $-0,13$ & $-0,04$ & 0,61 \\
\hline 02. Sinceridade & 0,78 & 0,01 & $-0,12$ & 0,07 & $-0,04$ & 0,54 \\
\hline 03. Fidelidade & $\mathbf{0 , 7 3}$ & $-0,09$ & $-0,08$ & 0,08 & 0,04 & 0,49 \\
\hline 12. Respeito & 0,72 & 0,10 & 0,09 & $-0,18$ & $-0,02$ & 0,55 \\
\hline 09. Amor & 0,56 & $-0,29$ & 0,22 & 0,12 & 0,10 & 0,46 \\
\hline 14. Diálogo & $\mathbf{0 , 3 2}$ & 0,22 & 0,14 & $-0,05$ & 0,17 & 0,44 \\
\hline 24. Conhecimento das intenções do outro & $-0,08$ & 0,63 & 0,05 & $-0,01$ & 0,07 & 0,42 \\
\hline 16. Coerência entre palavras e atos de parte a parte & $-0,07$ & 0,57 & 0,03 & $-0,06$ & 0,19 & 0,41 \\
\hline 25. Crédito de parte a parte à relação, acreditar nela & 0,17 & $\mathbf{0 , 5 3}$ & $-0,09$ & 0,12 & 0,08 & 0,50 \\
\hline 20. Preocupação igual consigo e com o outro & 0,14 & 0,51 & 0,08 & 0,05 & $-0,08$ & 0,43 \\
\hline 21. Capacidade de lidar com os problemas & 0,06 & $\mathbf{0 , 5 1}$ & 0,25 & $-0,09$ & $-0,10$ & 0,40 \\
\hline 19. Intimidade & $-0,14$ & $\mathbf{0 , 4 1}$ & $-0,10$ & 0,28 & 0,11 & 0,28 \\
\hline 26. Ausência de egoísmo & $-0,03$ & 0,35 & $-0,09$ & 0,15 & 0,14 & 0,21 \\
\hline 18. Empenho mútuo em manter a relação & 0,25 & 0,27 & 0,15 & 0,08 & 0,04 & 0,42 \\
\hline 11. Cooperação mútua & $-0,03$ & 0,03 & 0,76 & $-0,10$ & 0,10 & 0,60 \\
\hline 22. Construção compartilhada de algo & $-0,16$ & 0,09 & 0,67 & 0,17 & $-0,11$ & 0,48 \\
\hline 10. Planos compartilhados & $-0,03$ & $-0,05$ & 0,67 & 0,05 & 0,01 & 0,42 \\
\hline 17. Disposição mútua para enfrentar as dificuldades juntos & 0,14 & 0,14 & $\mathbf{0 , 5 0}$ & 0,06 & $-0,10$ & 0,46 \\
\hline 23. Sentimento mútuo de apoio & $-0,01$ & 0,32 & 0,38 & 0,08 & 0,01 & 0,48 \\
\hline 15. Troca, reciprocidade & 0,02 & 0,16 & 0,25 & 0,10 & 0,24 & 0,41 \\
\hline 29. Sentir a falta na ausência do outro & $-0,04$ & 0,00 & 0,04 & 0,63 & $-0,07$ & 0,36 \\
\hline 30. Externalização freqüente dos sentimentos de parte a parte & $-0,07$ & 0,03 & 0,12 & $\mathbf{0 , 5 9}$ & 0,00 & 0,41 \\
\hline 04. Encontros freqüentes e regulares & $-0,04$ & 0,03 & $-0,01$ & 0,46 & 0,13 & 0,28 \\
\hline 28. Busca contínua de satisfação do outro & 0,08 & 0,35 & $-0,10$ & 0,43 & $-0,07$ & 0,41 \\
\hline 05. Real envolvimento afetivo, ou vontade de se envolver & 0,14 & $-0,19$ & 0,05 & $\mathbf{0 , 3 8}$ & 0,36 & 0,41 \\
\hline 27. Estar juntos em eventos importantes da vida de cada um & 0,13 & 0,15 & 0,20 & 0,35 & $-0,15$ & 0,37 \\
\hline 07. Aceitação & 0,04 & 0,19 & $-0,23$ & 0,06 & 0,63 & 0,44 \\
\hline 08. Tolerância & $-0,09$ & 0,21 & 0,07 & $-0,08$ & 0,56 & 0,42 \\
\hline 06. Compreensão mútua & 0,06 & $-0,10$ & 0,31 & $-0,01$ & 0,48 & 0,46 \\
\hline 13. Flexibilidade & $-0,07$ & 0,30 & 0,24 & $-0,12$ & 0,33 & 0,42 \\
\hline Eigenvalue (Componentes Principais) & 9,95 & 1,94 & 1,45 & 1,25 & 1,19 & \\
\hline Eigenvalue (Análise Fatorial PAF) & 9,39 & 1,44 & 0,85 & 0,68 & 0,62 & \\
\hline Variância explicada & 33,15 & 6,46 & 4,84 & 4,16 & 4,00 & \\
\hline Variância explicada após rotação & 31,30 & 4,81 & 2,83 & 2,28 & 2,07 & \\
\hline
\end{tabular}

Nota: Saturações baseadas em uma Análise Fatorial Exploratória, adotando o método de extração dos eixos principais e com rotação promax (a rotação convergiu após sete interações). As saturações em destaque indicam os itens que foram selecionados para cada fator. Fator I - Comprometimento; Fator II - Edificação; Fator III - Mutualidade; Fator IV - Envolvimento; e Fator V - Indulgência.

sional sugerida no estudo de construção da RAE, decidiu-se realizar inicialmente duas novas AFEs. Inicialmente verificou-se que todos os itens apresentaram saturação maior do que 0,30 quando uma AFE foi realizada fixando a extração de um fator. Como no estudo de validação da escala a autora apresentou a possibilidade de uma estrutura bifatorial, realizou-se uma segunda AFE, adotando o método de extração dos eixos principais (PAF), rotação promax e fixando a extração de dois fatores. O primeiro fator foi composto por 24 itens $(22,11,23,13,24,15,16,08,10,19,30,06,25$, $17,21,20,07,28,18,04,27,26,05$ e 29$)$, enquanto apenas cinco itens saturaram no segundo fator $(01,02,03,12$ e 09$)$. $\mathrm{O}$ item 14 apresentou saturação superior a $0,30 \mathrm{em}$ ambos os fatores e foi excluído. Estes fatores foram denominados Amor Romântico e Amizade (Reis, 1995).

A partir dos resultados das AFEs, três modelos alternativos da estrutura fatorial da RAE foram testados através de AFCs. No Modelo 1, unifatorial, todos os itens foram especificados para apresentar saturação em um único fator. No Modelo 2, bidimensional, a estrutura fatorial descrita acima foi testada. Ou seja, os itens do primeiro fator foram fixados para saturarem em um fator (Amor Romântico), o mesmo acontecendo com os itens do segundo fator (Amizade). Por fim, o Modelo 3 testou a estrutura com cinco fatores (Comprometimento, Edificação, Mutualidade, Envolvimento e Indulgência), apresentados na Tabela 1. Os resultados das AFCs são sumarizados na Tabela 2.

Todas as saturações dos itens foram estatisticamente diferentes de zero $(t>1,96, p<0,05)$ para os três modelos. Como pode ser visto na Tabela 2 , todos os modelos apresentaram índices de bondade de ajuste razoáveis. Não obstante, enquanto o Modelo 2 foi estatisticamente superior ao Modelo $1\left[\Delta \chi^{2}(29)=971,42, p<0,001\right]$, foi inferior ao Modelo 3 $\left[\Delta \chi^{2}(111)=676,69, p<0,001\right]$. Desta forma, embora o Modelo 2 tenha apresentado uma pequena redução no SRMR, o Modelo 3 apresentou os melhores índices de bondade de ajuste. 
T. L. Milfont \& cols.

Tabela 2. Índices de bondade de ajuste dos modelos alternativos da Escala ERA.

\begin{tabular}{|c|c|c|c|c|c|c|c|c|c|}
\hline Modelo & $\chi^{2}$ & g.l. & $\chi 2 /$ g.l. & GFI & CFI & CAIC & $\begin{array}{c}\text { ECVI } \\
\text { (IC 90\%) } \\
\end{array}$ & $\begin{array}{l}\text { RMSEA } \\
\text { (IC 90\%) } \\
\end{array}$ & SRMR \\
\hline Modelo 1. Unifatorial & 2461,86 & 405 & 6,10 & 0,80 & 0,94 & 2911,21 & $\begin{array}{c}3,93 \\
(3,70 ; 4,17)\end{array}$ & $\begin{array}{c}0,088 \\
(0,085 ; 0,091)\end{array}$ & 0,063 \\
\hline Modelo 2. Bidimensional & 1490,44 & 376 & 4,00 & 0,86 & 0,96 & 1932,31 & $\begin{array}{c}2,45 \\
(2,27 ; 2,63)\end{array}$ & $\begin{array}{c}0,067 \\
(0,064 ; 0,071)\end{array}$ & 0,053 \\
\hline Modelo 3. Cinco fatores & 813,75 & 265 & 3,07 & 0,91 & 0,97 & 1263,10 & $\begin{array}{c}1,42 \\
(1,30 ; 1,56)\end{array}$ & $\begin{array}{c}0,056 \\
(0,052 ; 0,061)\end{array}$ & 0,054 \\
\hline
\end{tabular}

Notas: $\chi^{2}$ - qui-quadrado; g.l. - graus de liberdade; $\chi^{2} / g . l$ - razão do qui-quadrado pelo grau de iberdade; GFI - índice de bondade de ajuste; CFI - índice de ajuste comparativo; CAIC - critério de informação consistente de Akaike; ECVI - índice esperado de validação cruzada; $90 \% \mathrm{CI}$ - intervalo de consistência de $90 \%$ do ECVI; RMSEA - raíz quadrada média do erro de aproximação; 90\%CI - intervalo de consistência de 90\% do RMSEA; SRMR - raíz quadrada média residual estandartizada.

Os itens correspondentes a cada um dos cinco fatores foram computados para formar o escore fatorial das subescalas correspondentes. Estas apresentaram intercorrelações altas e significativas $(p<0,001)$, como apresentado na Tabela 3.

Como o modelo com cinco fatores (Modelo 3) foi o que apresentou os melhores parâmetros, corroborando a solução fatorial exploratória, decidiu-se testar um modelo hierárquico, ou modelo fatorial de segunda ordem (second-order factor model, Rindskopf \& Rose, 1988, Figura 1b). Neste modelo, os cinco fatores descritos anteriormente foram fixados como fatores de primeira ordem (first-order factors) e as atitudes frente a relações afetivas estáveis (RAE) como o fator de segunda ordem. Este modelo também apresentou índices de bondade de ajuste aceitáveis: $\chi^{2}(270)=817,84 ; \chi^{2} / g . l_{=}=3,03$; $G F I=0,91 ; C F I=0,97 ; C A I C=1229,74 ; E C V I=1,41 ; 90 \%$ $E C V I=1,29,1,55 ;$ RMSEA $=0,056 ; 90 \%$ RMSEA $=0,051$, 0,060; $S R M R=0,054$. O modelo hierárquico apresentou índices virtualmente idênticos e não foi estatisticamente superior ao Modelo $3\left[\Delta \chi^{2}(111)=676,69, p<0,001\right]$. A solução estandardizada desse modelo hierárquico é apresentada na Figura 1.

\section{Indicadores Complementares da Validade dos Cinco Fatores}

A validade convergente-discriminante dos cinco fatores foi avaliada por meio de correlações com duas variáveis externas: as pontuações dos participantes nas subescalas de auto-imagem Independente e Interdependente e os valores Pessoais, Centrais e Sociais (ver Tabela 3). Considerando-se a escala RAE como unidimensional, não há diferenciação entre as variáveis, pois correlações positivas e significativas foram encontradas com todas as cinco variáveis externas (auto-imagens e valores). Não obstante, considerando os cinco fatores (Comprometimento, Edificação, Mutualidade, Envolvimento e Indulgência), há a possibilidade de discriminação. Espeficicamente, Envolvimento e Indulgência não apresentaram correlações significativas com a auto-imagem Independente, enquanto que Edificação o fez com a autoimagem Interpedendente. Com relação aos valores, Comprometimento e Indulgência não apresentaram correlações significativas com os Pessoais.

Além destas correlações com variáveis externas, e diante do caráter exploratório da validade dos fatores encontrados, foram realizadas análises de variância (ANOVA) visando verificar se há diferença significativa nas médias dos sujeitos na RAE unifatorial e nos cinco fatores (Comprometimento,
Edificação, Mutualidade, Envolvimento e Indulgência), considerando-se as seguintes variáveis: importância da religião, com que freqüência vai a igreja (templo, sinagoga, etc.), situação de solteiro (se está sem ninguém, com namorado ocasional, namorando fixo ou noivo), opção sexual, e a forma que pensa legitimar a união (matrimônio civil-religioso, apenas matrimônio religioso, apenas matrimônio civil, ou

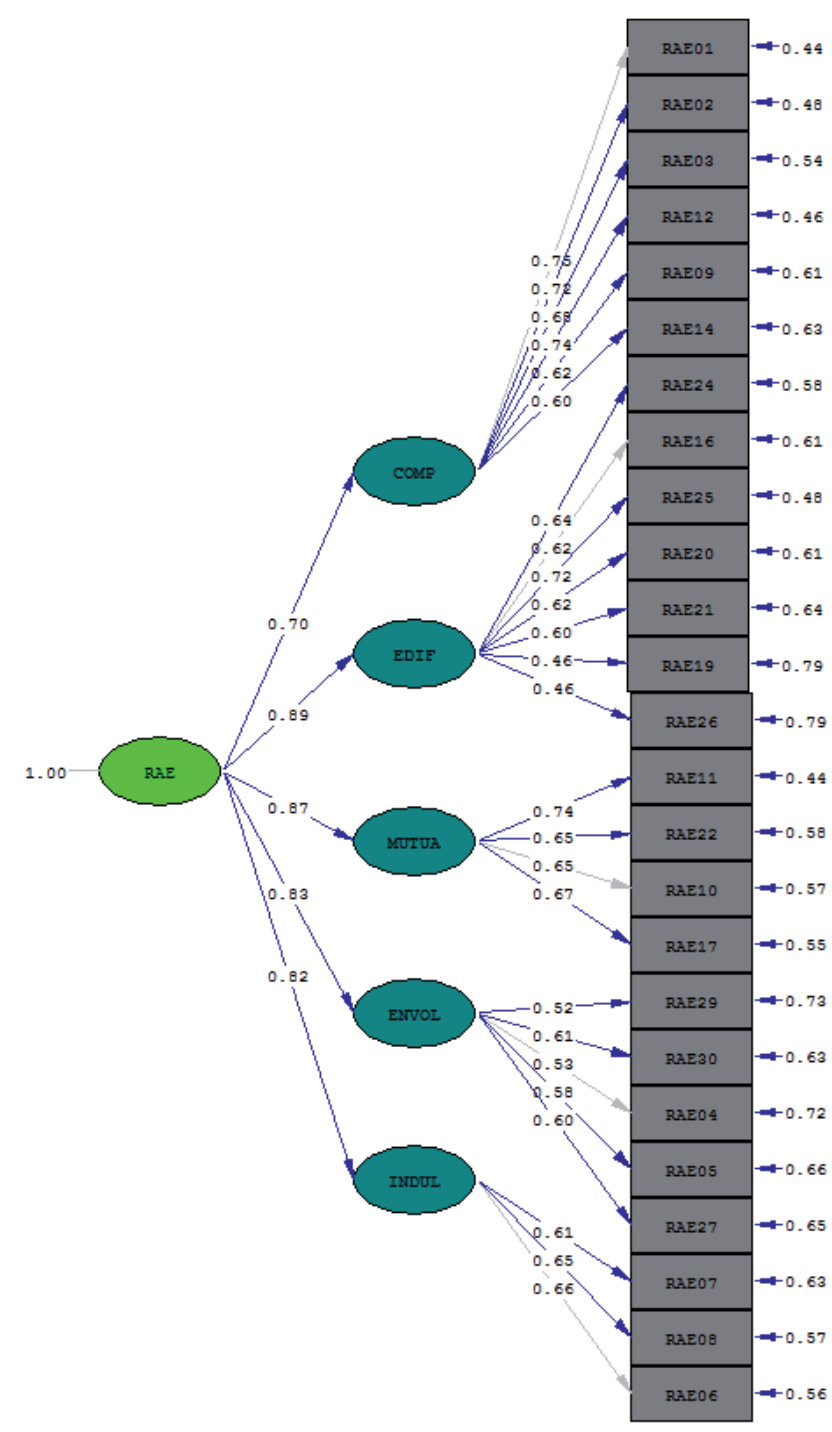

Figura 1. Solução estardartizada da análise fatorial confirmatória de segunda ordem da escala RAE $(N=658)$. 
Tabela 3. Índices de consistência interna e correlações da escala RAE e dos seus cinco fatores com as auto-imagens e valores humanos ( $N=658$ ).

\begin{tabular}{|c|c|c|c|c|c|c|c|c|c|c|c|c|c|c|}
\hline Sub-Escala & $\begin{array}{l}\text { № } \\
\text { Itens }\end{array}$ & M & DP & 1 & 2 & 3 & 4 & 5 & 6 & 7 & 8 & 9 & 10 & 11 \\
\hline 1. RAE unifatorial & 30 & 4,21 & 0,42 & $(0,93)$ & & & & & & & & & & \\
\hline 2. Comprometimento & 06 & 4,67 & 0,46 & $0,74 * * *$ & $(0,83)$ & & & & & & & & & \\
\hline 3. Edificação & 07 & 4,14 & 0,53 & $0,86^{* * *}$ & $0,52 * * *$ & $(0,77)$ & & & & & & & & \\
\hline 4. Mutualidade & 04 & 4,07 & 0,58 & $0,79 * * *$ & $0,50 * * *$ & 0,60 *** & $(0,77)$ & & & & & & & \\
\hline 5. Envolvimento & 05 & 3,95 & 0,56 & $0,77 * * *$ & $0,47 * * *$ & $0,55^{* * *}$ & $0,55^{* * * *}$ & $(0,71)$ & & & & & & \\
\hline 6. Indulgência & 03 & 4,13 & 0,58 & $0,70 * * *$ & $0,44 * * *$ & $0,55 * * *$ & $0,50^{* * *}$ & $0,45^{* * *}$ & $(0,68)$ & & & & & \\
\hline $\begin{array}{l}\text { 7. Auto-imagem } \\
\text { Independente }\end{array}$ & 15 & 4,83 & 0,60 & $0,13 * *$ & $0,09 *$ & $0,19 * * *$ & $0,10^{*}$ & 0,07 & 0,05 & $(0,50)$ & & & & \\
\hline $\begin{array}{l}\text { 8. Auto-imagem } \\
\text { Interdependente }\end{array}$ & 15 & 4,61 & 0,60 & $0,16^{* * *}$ & 0,07 & $0,14 * * *$ & $0,14 * * *$ & $0,15^{* * *}$ & $0,14 * * *$ & $-0,02$ & $(0,54)$ & & & \\
\hline 9. Valores Pessoais & 09 & 5,37 & 0,81 & $0,14 * * *$ & $-0,01$ & $0,22 * * *$ & $0,10^{*}$ & $0,14 * * *$ & 0,05 & $0,37 * * *$ & $-0,13^{* *}$ & $(0,58)$ & & \\
\hline 10. Valores Centrais & 07 & 6,15 & 0,62 & $0,36^{* * *}$ & $0,19 * * *$ & $0,34 * * *$ & $0,30 * * *$ & $0,27 * * *$ & $0,28 * * *$ & $0,22 * * *$ & $0,10^{*}$ & $0,35^{* * *}$ & $(0,46)$ & \\
\hline 11. Valores Sociais & 08 & 5,83 & 0,73 & $0,28 * * *$ & $0,22 * * *$ & $0,19 * * *$ & $0,29 * * *$ & $0,26^{* * *}$ & $0,16^{* * * *}$ & $-0,06$ & $0,31 * * *$ & $-0,07$ & $0,30 * * *$ & $(0,49)$ \\
\hline
\end{tabular}

Nota: ${ }^{*} p<0,05 ;{ }^{* *} p<0,01 ; * * * p<0,001$. Teste de significância bi-caudal. Os valores na diagonal correspondem aos Alfas de Cronbach das escalas.

Tabela 4. Teste $t$ entre a escala RAE e a variável sexo.

\begin{tabular}{llllllll}
\hline Escala & Sexo & Grau de Importância & M & DP & t & g.l. & p \\
\hline \multirow{2}{*}{ RAE unifatorial } & Homens & --- & 4,15 & 0,44 & & & \\
& Mulheres & --- & 4,29 & 0,39 & $-4,36$ & 656 & 0,001 \\
Comprometimento & Homens & $1^{\circ}$ & 4,57 & 0,50 & & & \\
& Mulheres & $1 \circ$ & 4,77 & 0,39 & $-5,61$ & 656 & 0,001 \\
Edificação & Homens & $2 \circ$ & 4,11 & 0,55 & & & \\
& Mulheres & $3 \circ$ & 4,19 & 0,51 & $-1,87$ & 656 & 0,062 \\
Mutualidade & Homens & $4 \circ$ & 4,00 & 0,61 & & & \\
\multirow{2}{*}{ Envolvimento } & Mulheres & $4 \circ$ & 4,16 & 0,54 & $-3,53$ & 656 & 0,001 \\
& Homens & $5 \circ$ & 3,86 & 0,59 & & & \\
Indulgência & Mulheres & $5 \circ$ & 4,05 & 0,52 & $-4,27$ & 656 & 0,001 \\
& Homens & $3 \circ$ & 4,06 & 0,60 & & & \\
& Mulheres & $2 \circ$ & 4,20 & 0,54 & $-3,23$ & 656 & 0,001 \\
\hline
\end{tabular}

Nota: Homens $(n=337)$, Mulheres $(n=321)$.

união livre, sem contrato legal algum). Os resultados demonstraram não haver diferença significativa $(p>0,05) \mathrm{em}$ nenhum destes grupos quanto ao escore na RAE unifatorial ou nos cinco fatores.

Além disso, foram efetuados testes $t$ de Student para verificar possíveis diferenças no escore médio dos seguintes grupos: homens e mulheres, os que pretendem ou não ter filhos e os que se imaginam ou não passando o resto da vida com uma mesma pessoa, na RAE unifatorial e nos cinco fatores. Os resultados mostraram diferenças significativas apenas no que se refere ao sexo. Como pode ser observado na Tabela 4 , houve diferenças significativas $(p<0,001)$ nas médias de homens $(M=4,15)$ e mulheres $(M=4,29)$ em relação à RAE unifatorial, como também em quatro dos cinco fatores. Apenas o fator Edificação não discriminou $(p>0,05)$ entre homens $(M=4,11)$ e mulheres $(M=4,19)$. Mas é interessante notar que as mulheres e os homens atribuíram quase o mesmo grau de importância a todos os cinco fatores (ver Tabela 4, coluna 3). Apenas para Indulgência as mulheres atribuíram mais importância do que os homens, enquanto que estes o fizeram em relação à Edificação.

\section{Discussão}

A atração interpessoal é um tema freqüente e importante em Psicologia Social, pois é um dos tópicos que apresentam mais respaldos empíricos nesta disciplina (Richard \& cols., 2003). Embora apresente várias áreas relacionadas, foi o estudo do amor que gerou o maior número de teorias e medidas. Não obstante, o ciúme e a avaliação das pessoas frente a relações afetivas estáveis também têm sido considerados. O presente estudo tratou exatamente sobre uma dessas áreas circundantes da atração interpessoal. Especificamente, o objetivo foi comprovar a estrutura fatorial da Escala de Atitudes Frente a Relacionamentos Afetivos Estáveis (RAE; Reis, 1995), testando sua validade e fidedignidade. Esta é uma das poucas medidas desenvolvidas no Brasil para a testagem de atitudes relacionadas à atração interpessoal.

Resultados de análises fatoriais exploratórias e confirmatórias indicaram que os itens da RAE apresentam uma estrutura multidimensional. Todos os cinco fatores encontrados (Comprometimento, Edificação, Mutualidade, Envolvimento e Indulgência) apresentaram índices de consistência interna 


\section{T. L. Milfont \& cols.}

satisfatórios e itens com saturações altas e significativas. Mais importante, esta estrutura multidimensional apresentou melhores índices de bondade de ajuste e foi estatisticamente superior do que a estrutura unidimensional, tal como sugerida pela sua autora (Bystronski, 1991; Reis, 1995).

Embora composta por outros itens e com um diferente formato ${ }^{4}$, alguns dos cinco fatores da RAE encontrados são coerentes com outros de pesquisas prévias. O primeiro fator, Comprometimento, é relacionado com um dos componentes do amor porposto por Stenberg (1986): decisão/comprometimento. Indulgência e Envolvimento são relacionados com os fatores respeito e intimidade comunicativa, tal como medido pela escala da intensidade dos componentes do amor (Hernandez \& Oliveira, 2003). Ainda, Mutualidade assemelha-se com um dos estilos secundários de amor (pragma - amor racional, lógico) proposto por Lee (1977).

Com relação à validade dos cinco fatores, os resultados demonstraram que eles são mais úteis do que a estrutura unidimensional. Inicialmente, verificou-se que para a autoimagem Independente e os valores Pessoais e Sociais, alguns dos cinco fatores apresentaram índices de correlação mais altos do que o observado, ao considerar a RAE como unifatorial (ver Tabela 3). Além disso, considerando-se a escala RAE como unidimensional, esta apresenta correlações positivas e significativas com todas as cinco variáveis externas consideradas. Por outro lado, nem todos os cinco fatores apresentaram correlações significativas com as auto-imagens ou os valores.

Os fatores Envolvimento e Indulgência não apresentaram correlações significativas com a auto-imagem Independente, indicando talvez serem incompatíveis com uma visão autônoma do self, mas sim mais compatíveis com uma visão relacional do self (por isso, Envolvimento apresentou a maior correlação com a auto-imagem Interdependente). Em contraste, Comprometimento foi o único fator a não se correlacionar com a autoimagem Interdependente. Embora se refira ao compromisso com o relacionamento afetivo que se tem ou que se planeja ter, este fator indica uma maior necessidade de autonomia do self. As correlações deste fator com os valores também suportam esta idéia. Apenas Comprometimento e Indulgência não apresentaram correlações significativas com os valores Pessoais.

Análises de variância (ANOVA) e testes $t$ de Student também foram realizados para verificar possíveis diferenças da RAE unifatorial e dos cinco fatores em relação a uma série de variáveis. Por exemplo, esperava-se que as pessoas com um relacionamento afetivo duradouro apresentariam escores significativamente maiores do que os que estavam solteiros no momento da pesquisa. Não obstante, apenas diferenças em relação ao sexo foram encontradas. Condizentes com os achados de Reis (1995), as mulheres apresentaram escores maiores do que os homens na RAE unidimensional, e em quatro dos cinco fatores. Apenas para o fator Edificação não houve diferença. Composto pelos itens conhecimento das intenções do outro, coerência entre palavras e atos de parte a parte, crédito de parte a parte à relação, preocupação consigo e com o outro, capacidade de lidar com os problemas, intimidade e ausência de egoísmo, este fator refere-se

4 Os itens da RAE são substantivos (por exemplo, confiança) ou pequenas construções (por exemplo, externalização freqüente dos sentimentos de parte a parte), enquanto que os itens de outras escalas são geralmente sentenças mais longas. a aspectos importantes na construção de uma relação afetiva estável. Embora em geral os homens tenham atribuído um maior peso a este fator do que as mulheres (como apresentado na Tabela 4, coluna 2), Edificação aparenta ser um aspecto importante e com igual peso para universitários e universitárias em uma relação afetiva estável.

Em conjunto, os resultados do presente estudo suportam a validade e fidedignidade da Escala de Atitudes frente a Relações Afetivas Estáveis (RAE). As características psicométricas foram satisfatórias e a estrutura multidimensional aparenta ser mais robusta e útil do que a estrutura unidimensional. No presente estudo, as pessoas que já possuíam um relacionamento afetivo duradouro não apresentaram escores significativamente maiores do que os que estavam solteiras no momento da pesquisa, tal como era teoricamente esperado (Reis, 1995). Desta forma, em estudos futuros recomenda-se que esta hipótese e os cinco fatores aqui encontrados sejam novamente testados com uma amostra diferenciada, já que as pesquisas prévias e a presente foram realizadas apenas com estudantes universitários, e que sejam criados novos itens para o fator Indulgência, pois foi composto apenas por três itens.

\section{Referências}

Alferes, V. R. (1996). Atracção interpessoal, sexualidade e relações íntimas. Em J. Vala \& M. B. Monteiro (Orgs.), Psicologia social ( $2^{\mathrm{a}}$ ed.) (pp. 115-139). Lisboa: Fundação Calouste Gulbenkian.

Aronson E., Wilson, T. D. \& Akert, R. M. (2002). Psicologia social. Rio de Janeiro: LTC.

Baron, R. A. \& Byrne, D. (2002). Social psychology (10 ed.). Boston: Allyn and Bacon.

Belo, R. P., Raymundo, J. S., Gouveia, V. V., Milfont, T.L. \& Vasconcelos, T. C. (2002). Validação de uma versão reduzida da Escala de Ciúme Romântico. [Resumo]. Em Conselho Federal de Psicologia (Org.), Resumos de comunicações científicas, I Congresso Brasileiro de Psicologia: Ciência e Profissão, São Paulo.

Bystronski, B. (1991). A liberação dos costumes e suas conseqüências sobre os relacionamentos amorosos heterossexuais. Dissertação de Mestrado, Universidade Federal do Rio Grande do Sul, Porto Alegre.

Carmines, E. G. \& McIver, J. D. (1981). Analyzing models with unobserved variables: Analysis of covariance structures. Em G. W. Bohinstedt \& E. F. Borgatta (Orgs.), Social measurement: Current issues (pp. 65-115). Beverly Hills: Sage.

Fabrigar, L. R., MacCallum, R. C., Wegener, D. T. \& Strahan, E. J. (1999). Evaluating the use of exploratory factor analysis in psychological research. Psychological Methods, 4, 272-299.

García, C. Y. (2000). El amor desde la psicología social. Madri: Pirámide.

Garson, G. D. (2003). PA 765 Statnotes: An online textbook. Retirado em 24/11/2004 de http://www2.chass.ncsu.edu/garson/ pa765/statnote.htm.

Gouveia, V. V. (1998). La natureza de los valores descriptores del individualismo y del colectivismo: Una comparación intra e intercultural. Tese de Doutorado, Universidade Complutence de Madri, Espanha.

Gouveia, V. V. (2003). A natureza motivacional dos valores humanos: Evidências acerca de uma nova tipologia. Estudos de Psicologia, 8, 431-443. 
Gouveia, V. V., Singelis, T. M. \& Coelho, J. A. P. M. (2002). Escala de Auto-Imagem: Comprovação da sua estrutura fatorial. Avaliação Psicológica, 1, 49-59.

Hendrick, C. \& Hendrick, S. (1986). A theory and method of love. Journal of Personality and Social Psychology, 50, 392-402.

Hernandez, J. A. E. \& Oliveira, I. M. B. (2003). Os componentes do amor e a satisfação. Psicologia: Ciência e Profissão, 21, 58-69.

Hewstone, M. \& Strobe, W. (Orgs.) (2001). Introduction to social psychology: An European perspective ( $3^{\mathrm{a}}$ ed.). London: Blackwell.

Hu, L.-t. \& Bentler, P. M. (1999). Cutoff criteria for fit indexes in covariance structure analysis: Conventional criteria versus new alternatives. Structural Equation Modeling, 6, 1-55.

Jöreskog, K. G. \& Sörbom, D. (1996). LISREL 8: User's reference guides. Chicago: Scientific Software International.

Jöreskog, K. \& Sörbom, D. (1993). LISREL 8: Structural equation modeling with Simplis command language. Chicago, IL: Scientific Software International.

Laros, J. A. (2004). O uso da análise fatorial: Algumas diretrizes para pesquisadores. Em L. Pasquali (Org.), Análise fatorial para pesquisadores (pp. 147-170). Petrópolis: Vozes.

Lee, J. A. (1977). A typology of styles of loving. Personality and Social Psychology Bulletin, 3, 173-182.

Milfont, T. L. (no prelo). Estrutura fatorial da Escala de AutoImagem: Testando modelos alternativos. Estudos de Psicologia (PUCCAMP).

Myers, D. G. (2000). Psicologia social. Rio de Janeiro: LTC.

O'Connor, B. P. (2000). SPSS, SAS, and MATLAB programs for determining the number of components using parallel analysis and Velicer's MAP test. Behavior Research Methods, Instruments, \& Computers, 32, 396-402.

Ramos, A. L. M., Yazawa, S. A. K. \& Salazar, A. F. (1994). Desenvolvimento de uma escala de ciúme romântico. Psicologia: Teoria e Pesquisa, 10, 439-451.

Reis, B. F. (1992). O amor à luz da psicologia científica. Psicologia: Reflexão e Crítica,_5, 23-40.

Reis, B. F. (1995). Uma escala de atitudes frente a relações afetivas estáveis. Psicologia: Teoria e Pesquisa, 11, 67-71.
Richard, F. D., Bond C. F., Jr. \& Stokes-Zoota, J. J. (2003). One hundred years of social psychology quantitatively described. Review of General Psychology, 7, 331-363.

Rindskopf, D. \& Rose, T. (1988). Some theory and applications of confirmatory second order factor analysis. Multivariate Behavioral Research, 23, 51-67.

Rodrigues, A., Assmar, E. M. L. \& Jablonski, B. (2001). Psicologia social. Petrópolis: Vozes.

Rubin, Z. (1970). Measurement of romantic love. Journal of Personality and Social Psychology, 16, 265-273.

Schafer, J. L. (1997). Analysis of incomplete multivariate data. London: Chapman \& Hall.

Schafer, J. L. \& Graham, J. W. (2002). Missing data: Our view of the state of the art. Psychological Methods, 7, 147-177.

Singelis, T. M. (1994). The measurement of independent and interdependent self-construals. Personality and Social Psychology Bulletin, 20, 580-591.

Sinharay, S., Stern, H. S. \& Russell, D. (2001). The use of multiple imputation for the analysis of missing data. Psychological Methods, 6, 317-329.

Smith, P. B. \& Bond, M. H. (1993). Social psychology across cultures: Analysis and perspectives. New York: Harvester Wheatsheaf.

Stenberg, R. J. (1986). A triangular theory of love. Psychological Review, 93, 119-135.

Sternberg, R. J. (1997). Construct validation of a triangular love scale. European Journal of Social Psychology, 27, 313-335.

Ubillos, S., Zubieta, E., Páez, D., Deschamps, J., Ezeiza, A. \& Vera, A. (1997). Amor, cultura y sexo. Revista Electrónica de Motivación y Emoción, 4. Retirado em 24/11/2004 de http:// reme.uji.es/articulos/aubils9251701102/texto.html.

Vaughan, G. M. \& Hogg, M. A. (2002). Introduction to social Psychology (3a ed.). Frenchs Forest: Prentice Hall.

Recebido em 07.07.2005

Primeira decisão editorial em 24.08.2006

Versão final em 17.04.2007

Aceito em 14.08.2008 